\title{
Anterior cruciate ligament- specialized post-operative return-to-sports (ACL-SPORTS) training: a randomized control trial
}

\author{
Kathleen White ${ }^{1 *}$, Stephanie L Di Stasi ${ }^{2}$, Angela H Smith ${ }^{3}$ and Lynn Snyder-Mackler ${ }^{1,3}$
}

\begin{abstract}
Background: Anterior cruciate ligament reconstruction (ACLR) is standard practice for athletes that wish to return to high-level activities; however functional outcomes after ACLR are poor. Quadriceps strength weakness, abnormal movement patterns and below normal knee function is reported in the months and years after ACLR. Second ACL injuries are common with even worse outcomes than primary ACLR. Modifiable limb-to-limb asymmetries have been identified in individuals who re-injure after primary ACLR, suggesting a neuromuscular training program is needed to improve post-operative outcomes. Pre-operative perturbation training, a neuromuscular training program, has been successful at improving limb symmetry prior to surgery, though benefits are not lasting after surgery. Implementing perturbation training after surgery may be successful in addressing post-operative deficits that contribute to poor functional outcomes and second $A C L$ injury risk.

Methods/Design: 80 athletes that have undergone a unilateral ACLR and wish to return to level 1 or 2 activities will be recruited for this study and randomized to one of two treatment groups. A standard care group will receive prevention exercises, quadriceps strengthening and agility exercises, while the perturbation group will receive the same exercise program with the addition of perturbation training. The primary outcomes measures will include gait biomechanics, clinical and functional measures, and knee joint loading. Return to sport rates, return to pre-injury level of activity rates, and second injury rates will be secondary measures.
\end{abstract}

Discussion: The results of this ACL-Specialized Post-Operative Return To Sports (ACL-SPORTS) Training program will help clinicians to better determine an effective post-operative treatment program that will improve modifiable impairments that influence outcomes after ACLR.

Trial registration: Randomized Control Trial NIH 5R01AR048212-07. ClinicalTrials.gov: NCT01773317

Keywords: Anterior cruciate ligament reconstruction, Neuromuscular training, Return to Sport

\section{Background}

Anterior cruciate ligament reconstruction (ACLR) is standard practice for individuals that desire to return to high-level activities, but excellent outcomes are not as commonplace as previously reported [1-5]. Currently, success after ACLR is measured using return-to-sport rates, but second ACL injuries are not only common, but devastating, and have worse outcomes than primary ACLR [6-8]. Quadriceps weakness [9-11], abnormal movement

\footnotetext{
* Correspondence: katwhite@udel.edu

'University of Delaware, Biomechanics and Movement Science Program, Newark, DE, USA

Full list of author information is available at the end of the article
}

patterns [4,12-16] and below normal knee function [17] are characteristic of athletes in the months following ACLR and often persist up to two years in spite of extensive rehabilitation. Neuromuscular training focusing on restoring limb symmetry and improving knee function using sports-related movements may reduce aberrant movement patterns which are predictive of second injury risk $[4,12,18,19]$.

Risk of a second ACL injury is highest during the first year that athletes return to sports after primary ACL reconstruction [4,13,20-22]. Young females are 16 times more likely to sustain a second ACL injury after primary ACLR and the amount of participation time in high-level

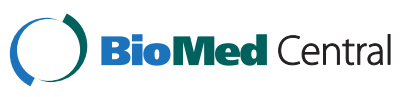


activities further increases this risk [23]. Risk to the contralateral limb is higher (5-24\%) than the operated limb (4-15\%) [4,23-28], suggesting deficits of the involved limb are not exclusively related to re-injury. Altered neuromuscular and biomechanical movement patterns are present bilaterally in response to injury and reconstruction which fails to resolve with post-operative rehabilitation. A neuromuscular training program focused on maximizing performance after ACLR may reduce the risk of a second ACL injury.

Despite current evidence-based post-operative guidelines [29-32], quadriceps strength deficits [9-11], altered biomechanics [4,13-16,33] and poor knee function [17] are reported six months and one year after surgery. International Knee Documentation Committee 2000 subjective knee form (IKDC 2000) scores continue to improve up to one year after surgery suggesting optimal knee function has not been met [17]. Despite clearance for return to sport activities by surgeons and rehabilitation specialists, quadriceps strength deficits of the involved limb compared to the uninvolved limb still exist, and movement asymmetries continue to persist $[10,11,14,16]$.

One year after surgery only $67 \%$ of patients have attempted some sort of training or sport activity; males are more likely than females to attempt full return to sport [34]. Individuals often do not return to their pre-injury activity level for a variety of reasons; fear of re-injury being a large contributing factor [35-37]. Patients in the medium to long term after surgery (two to seven years) that have returned to their pre-injury activity level were less likely to be fearful of re-injury during athletic participation than those that had not returned to their pre-injury level [37]. Females were more fearful with poor environmental conditions during athletic participation than their male counterparts [37].

Neuromuscular training, consisting of destabilizing perturbations to both the involved and uninvolved lower extremities, has been an effective means of enhancing functional outcomes after ACL injury compared to strength training $[12,38]$. Neuromuscular training programs such as perturbation training (PERT) [39] before surgery reduce gait asymmetries in female non-copers [18]. After surgery, non-copers who received pre-operative PERT demonstrated improved knee excursion symmetry during gait compared to patients who received strength training [12]. However, regardless of pre-operative intervention, aberrant movement patterns persisted up to two years after surgery [14]. Pilot data from our lab strongly suggests that utilizing this neuromuscular training program after surgery will be an effective means of improving both short term outcomes (6 months), when clearance to return to sport often occurs, and medium term outcomes (1-2 years) after surgery. Successful primary ACL prevention programs utilize a combination of balance, plyometric and strengthening exercises to decrease ACL injury risk. Similarly, our ACL-Specialized Post-Operative Return To Sports (ACL-SPORTS) Training will incorporate dynamic prevention exercises and quadriceps strengthening exercises that promote symmetrical joint loading and abate abnormal movement patterns. A post-operative intervention incorporating these elements with the addition of PERT may be effective in resolving residual impairments after surgery.

The purpose of this study is to determine the effects of this ACL-SPORTS Training program on joint loading, biomechanics, and clinical and functional measures of level 1 and 2 athletes after ACLR. This body of work will further explain in detail each component of the training program as well as the methodology of this single blinded randomized control trial.

\section{Hypotheses}

Subjects who receive standard care plus PERT after surgery will demonstrate: 1) symmetrical knee joint loading, 2) symmetrical movement patterns, 3) improved clinical and functional outcomes and 4) improved knee function compared to subjects who receive standard care. Additionally, subjects who receive standard care plus PERT will have a higher return to pre-injury level rates in the short to medium term (6 months -2 years) compared to subjects who receive standard care.

\section{Methods/Design}

This study is a single-assessor blinded, parallel design randomized control trial that follows the CONSORT guidelines for non-pharmacological treatment studies [40]. Additional information about this study can be found at: Clinicaltrials.gov (Identifier: NCT 01773317).

\section{Participants}

Eighty level 1 and 2 athletes (40 men, 40 women) between the ages of 13 and 55 that have undergone an isolated, unilateral ACL reconstruction will be recruited for this study. Recruiting will be done primarily through the University of Delaware Physical Therapy Clinic. Additional recruitment will consist of newspaper advertisements as well as speaking with local surgeons and rehabilitations specialists. Athletes will be eligible for study enrollment if they were participants in level 1 or 2 activities [1] $\geq 50 \mathrm{hrs} /$ year at the time of their injury, plan to return to their preinjury level of activity, are $\geq 12$ weeks after surgery, demonstrate $\geq 80 \%$ quadriceps strength index and minimal knee joint effusion [41].

\section{Exclusion criteria}

Subjects will be excluded if: (i) not regular participants in level 1 or 2 activities (<50 hrs/yr), (ii) $>10$ months after ACLR, (iii) history of previous ACLR, (iv) history of serious ipsilateral or contralateral limb injury (i.e. Tibial fx), 
or (v) large osteochondral defect $>1 \mathrm{~cm}^{2}$ (Figure $1 . \mathrm{CON}$ SORT Flow Diagram of Study Protocol).

\section{Procedure}

Once a patient has consented to be contacted by research staff, eligibility will be determined from patient chart review and patient dialogue. All testing measures will be performed at the University of Delaware Physical Therapy Clinic by the same assessor, blinded to group assignment. Enrolled study participants will complete initial baseline testing and 10 treatment sessions followed by posttraining, 1 year and 2 year follow-up testing sessions. Testing will be completed no more than two weeks prior to initiation and two weeks after the completion of the 10 training sessions. Ethical approval has been obtained from the University of Delaware Human Subjects Review board.
All participants will provide written informed consent to all research testing procedures.

\section{Randomization and allocation concealment}

Enrolled subjects will be randomized to a perturbation treatment group (PERT) or a standard treatment group (STND). A statistical random number generator will be used to generate a randomization list, stratified by gender in which an equal number of female and male subjects will be assigned to each treatment group. The research coordinator will generically label the treatment assignments to group A and B to ensure blinding is maintained. This is a single-blinded study in that individuals collecting, recording and analyzing these data will be blinded to group assignment along with the primary investigator and biostatistician. Both the treating

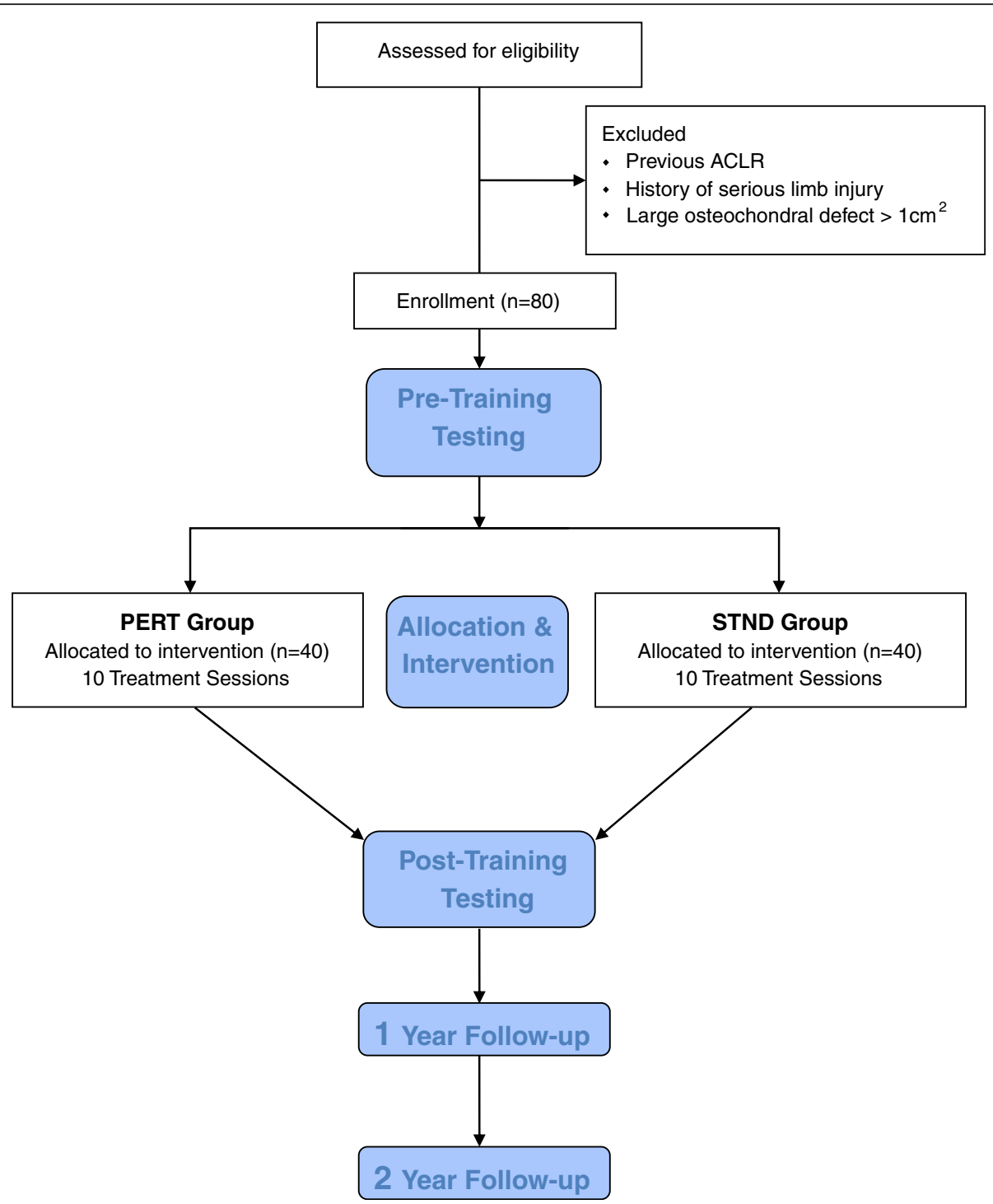

Figure 1 CONSORT flow diagram of study proctol. 
physical therapist and the patient will not be blinded to group assignment.

\section{Interventions}

Treatment will be completed at the University of Delaware Physical Therapy clinic by the licensed staff therapists. The therapists have an average of 6.5 yrs experience (range 1 20 yrs). All therapists will participate in a training session involving discussion of the treatment program and how to manage changes in effusion, complaints of muscle soreness and still effectively complete the training program. A detailed protocol with visual aids and descriptions will be provided. Once training has been initiated procedural reliability will be completed by an unblinded investigator to ensure that the intervention is properly executed. An unmasked physical therapy clinic liaison will utilize a procedural reliability check list to review three treatment sessions for the first five subjects in each arm of the study. After that each subject's chart will be reviewed once (Additional file 1). Procedural reliability less than $85 \%$ will result in contacting the treating therapist to remedy the situation. Any additional questions regarding the training program will be intercepted by the clinic liaison to ensure blinding of those responsible for data collection is maintained.

The training protocol consists of 10 training sessions with treatment delivered by a licensed physical therapist, regardless of group allocation. Biomechanical and functional data will be collected prior to initiation of the 10 training sessions, after completion of the sessions, and 1 and 2 years after surgery. The training program consists of a series of "prevention exercises", quadriceps strengthening exercises, agility drills and either PERT training for individuals in the perturbation group or a control exercise for individuals in the STND care group. Details of these exercises are explained in further detail below.

Prevention exercises: A combination of balance, plyometric and strengthening exercises are effective in preventing initial ACL injury $[30,42]$. Plyometric training improves landing biomechanics in females [43] and decreases ACL injury rates [44]. Balance training not only improves lower extremity strength, but eliminates limb asymmetries [45], which are potential risk factors for ACL injury $[4,46]$. Strengthening programs alone do not reduce the number of ACL injuries [47], however when combined with plyometric training there is a significant reduction in ACL injuries, specifically female athletes [44]. Established injury prevention protocols were modified to develop the "prevention exercises" for this study (Table 1). Plyometric and balance exercises include triple single-legged hops, tuck jumps and box drops; strengthening exercises include nordic hamstring curls and squats with hip abduction resistance. Triple singlelegged hops are done consecutively, forward and backwards as well as laterally. The initial drill will be completed by hopping over a line on the floor and progressed to hopping over 2 inch cups and ultimately 6 inch hurdles. Progression and cueing will be given by the treating therapist as per the patient's ability level with a training protocol as a guide (Table 1). Tuck jumps will not be completed until treatment sessions 7-10 to ensure that patients are able to tolerate jump landings safely and successfully. Box drops begin bilaterally and progress to unilateral jumps (involved limb to involved limb). Focus on mechanics during double limb tasks initially allows for an effective progression to single limb tasks [48]. The box height will be progressively increased by the treating therapist as per the patient's ability level using the training protocol as a guide (Table 1). This task will be completed in front of a mirror for visual feedback while the therapist provides verbal cues. Proper mechanics will be required including symmetrical limb takeoff and landing for bilateral tasks, good trunk control and neutral frontal plane knee alignment during takeoff and landing for bilateral and unilateral tasks. Nordic hamstring exercises will be completed on a low mat table with the therapist stabilizing the patient's ankles. Initially this eccentric hamstring activity will be done to about 30-45 degrees of knee flexion and repetitions as well as knee flexion angle will be progressed over the 10 training sessions. Resisted squat exercise will be done with a thera-band around the patient's knees to facilitate hip abduction. The resistance of the thera-band will increase as tolerated by the patient and additional upper extremity tasks such as a ball toss will be added to increase the difficulty of the task and challenge the patient. These exercises will be executed with the patient wearing a rigid functional knee brace if one has been prescribed by the surgeon. If a patient will be returning to sport without a knee brace then all testing and training sessions will be done without a brace.

Quadriceps strengthening: The results of baseline testing measures will be used to determine the patient's need for quadriceps strengthening during the 10 training sessions. A patient that demonstrates $>90 \%$ quadriceps strength index (involved limb strength/uninvolved limb strength $\times 100$ ) will not be required to complete quadriceps strengthening exercises during training, but they may continue their prior gym program. All other patients with $80-90 \%$ quadriceps strength index will complete three quadriceps strengthening exercises during three of the first six training sessions including but not limited to, lateral step downs, leg press, LAQ and isokinetic strengthening. After the $6^{\text {th }}$ training session the patient will be given a home strengthening program because of the progressive nature of the program and the advanced level of tasks during the last 4 sessions.

Agilities: Agility drills will be completed as per the University of Delaware guidelines. Drills will be initiated 
Table 1 ACL-SPORTS training protocol (2 times/wk)

\begin{tabular}{|c|c|c|c|c|}
\hline & Session 1-3 & & Session 4-6 & Session 7-10 \\
\hline Nordic Hamstrings & Partial $2 \times 5$ & & Partial $3 \times 5$ & Partial $3 \times 5$ \\
\hline $\begin{array}{l}\text { Kneeling on mat table, therapist } \\
\text { stabilizing feet }\end{array}$ & $\left(\sim 30-45^{\circ}\right)$ & & $\left(\sim 30-45^{\circ}\right)$ & $\left(>60^{\circ}\right)$ \\
\hline Standing Squat & \multirow{2}{*}{$\begin{array}{l}\text { Session 1: } 3 \times 10 \text { with focus } \\
\text { on proper technique }\end{array}$} & $3 \times 10$ & $3 \times 10$ & \\
\hline $\begin{array}{l}\text { Must squat to knees at } 90 \text { degrees, } \\
\text { tapping chair/table/box with gluts }\end{array}$ & & $\begin{array}{l}\text { Add t-band } \\
\text { around knees }\end{array}$ & $\begin{array}{l}\text { progress t-bands } \\
\text { to black }\end{array}$ & $x$ \\
\hline Drop jumps** & $3 \times 10$ BLE's to BLE's & & $3 \times 10$ BLE's to involved & $3 \times 10$ Involved limb to involved limb \\
\hline \multirow{2}{*}{$\begin{array}{l}\text { In front of mirror, monitor proper } \\
\text { form with landing }\end{array}$} & \multirow{2}{*}{\multicolumn{2}{|c|}{$\begin{array}{l}\text { Jump off appropriate } \\
\text { height (4-6-8 inch) }\end{array}$}} & $\operatorname{limb}$ & off box \\
\hline & & & $\begin{array}{l}\text { Jump off appropriate } \\
\text { height (4-6-8 inch) }\end{array}$ & Jump off appropriate height (4-6-8 inch) \\
\hline \multirow[t]{3}{*}{ Triple single leg hopping** } & \multicolumn{2}{|l|}{ Forward/backward $\times 10^{*}$} & Forward/backward x15* & Forward/backward x15* \\
\hline & \multicolumn{2}{|l|}{ Side to side $\times 10^{*}$} & Side to side $\times 15^{*}$ & Side to side $\times 15^{*}$ \\
\hline & \multirow{2}{*}{\multicolumn{2}{|c|}{ No object }} & $\begin{array}{l}\text { Add low object to } \\
\text { jump over ( } 2 \text { inch cups) }\end{array}$ & $\begin{array}{l}\text { Increase height of object, appropriate } \\
\text { for the pt. ( } 4 \text { inch cups or } 6 \text { inch hurdles) }\end{array}$ \\
\hline $\begin{array}{l}\text { This is for proper landing, NOT } \\
\text { distance }\end{array}$ & & & & \\
\hline \multicolumn{3}{|l|}{ Tuck jumps** } & & 2 sets, $10-20 \mathrm{sec}$ \\
\hline Proper form knees to $90^{\circ}$ & \multicolumn{2}{|l|}{$x$} & $x$ & Progress to 3 sets, $20-30$ seconds each \\
\hline
\end{tabular}

**Bace worn if surgeon requires post-op functional brace for RTS activities. * 1 rep $=3$ consecutive hops forward, 3 hops backward or 3 consecutive hops laterally.

at $50 \%$ maximum effort and progressed to $100 \%$ effort and maximum speed over the 10 training sessions. Three to four agilities drills will be completed at each training session including forward/backward running, side shuffles, cariocas, figure eight's, circles and 90 degree turns. The treating therapist will determine which agility drills to use based on the patients sports participation and ability level. Progression of these drills will include eliminating linear drills, adding more advanced multidirectional drills and utilizing a ball consistent with the patient's sport of participation.

Perturbation training group: Patients randomized to the PERT group will complete additional PERT training as per Fitzgerald et al. [38]. PERT training is a neuromuscular training program that includes a series of progressive perturbations on unstable surfaces in both bilateral and unilateral stance. These are progressed as per patient tolerance in both magnitude and speed. Verbal distraction as well as the addition of simultaneous upper extremity or lower extremity tasks with perturbations will be used to target the individuals sport and challenge the athlete.

Standard treatment group: Patients in the STND group will complete an additional single leg balance task with added hip flexor resistance (Figure 2). This exercise will not be progressed to unstable surfaces to ensure that similar neuromuscular effects are not seen in this group. This exercise will only increase in duration and theraband resistance (Table 2). All treatment sessions, regardless of group, will take about 1.5-2 hours to complete.

The expertise of the treating therapists will determine if any task is unsafe for the patient and should be held from the protocol at any point. If a patient develops increased knee joint effusion [41] or additional complaints of pain, a clinical decision making protocol has been established as a guide for the therapists to determine how to modify the training program (Figure 3 ). If a patient present with a $2+$ effusion at any point during the training program the training protocol will be held and the patient will be treated with effusion management including retrograde massage, ice and elevation. The

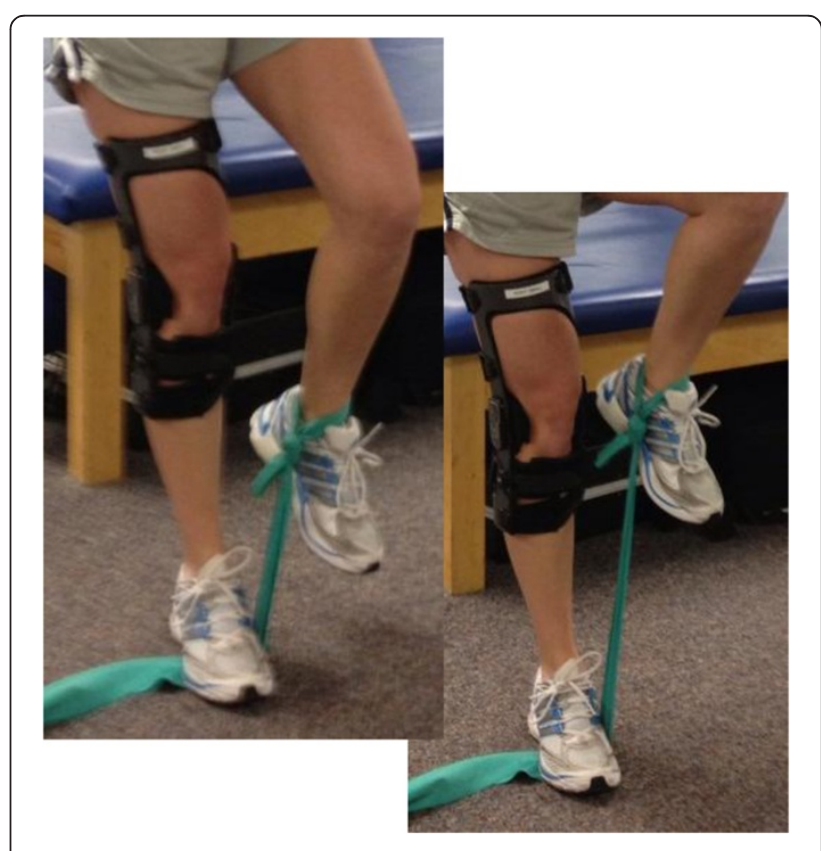

Figure 2 STND Treatment Group Additional Exercise. 
Table 2 STND treatment group additional exercise

\begin{tabular}{|c|c|c|c|}
\hline Control subjects & $\begin{array}{l}\text { Session } \\
1-3\end{array}$ & Session 4-6 & $\begin{array}{l}\text { Session } \\
7-10\end{array}$ \\
\hline Single Leg Balance & $3 \times 30 \mathrm{sec}$ & $3 \times 45 \mathrm{sec}$ & $\begin{array}{l}3 x \\
1 \text { minute }\end{array}$ \\
\hline $\begin{array}{l}\text { (Do Not "progress" with } \\
\text { unstable surfaces or } \\
\text { ball toss or perturbation) }\end{array}$ & $\begin{array}{l}\text { (Level } \\
\text { Ground) }\end{array}$ & $\begin{array}{l}\text { Add sham progression: } \\
\text { (stabilize t-band with } \\
\text { standing leg, complete } \\
\text { hip flexion with tband } \\
\text { around ankle) }\end{array}$ & same \\
\hline
\end{tabular}

patient will be educated on proper effusion management techniques such as keeping the knee wrapped with a donut and compression wrap (Figure 4), as well as icing several times a day with the leg elevated. At the following session if the effusion has decreased to a $1+$ the training will resume at the same level of difficulty, if the patient has trace or no effusion then the training program will be progressed accordingly. Conversely, if the patient continues to demonstrate a $2+$ effusion the training will be held, the patient will be treated accordingly for effusion and the research team will be notified. Additional complications that occur throughout training will be treated as needed by the treating therapist. If additional symptoms or impairments are limiting completion of the training program the principle investigator will be notified. If the patient cannot resume the training program for any reason the training will be terminated and post-training data will be collected. The patient will continue to be treated accordingly for their impairments.

\section{Outcomes measures}

The primary outcome variables of interest for this study will include gait biomechanics, clinical outcome measures and knee joint loading.

Gait biomechanics will be assessed using a 3D motion capture system (VICON, Oxford Metrics Ltd., London, England) sampled at $120 \mathrm{~Hz}$. Twenty static retro-reflective markers will be placed on the pelvis and lower extremities to identify limb segments. An embedded force plate (Bertec, Worthington, OH) will simultaneously collect kinetic data and used to determine timing variables during the gait cycle. Five walking trials will be collected for each limb while patients maintain a self-selected walking speed with $\pm 5 \%$ variability. These data will be post-processed using rigid body analysis and inverse dynamics with custom software programming (Visual3D, C-Motion, Inc., Germantown, MD, USA; LabVIEW 8.2, National Instruments Corp., Austin, TX, USA). Variables will be lowpass filtered at $6 \mathrm{~Hz}$ and $40 \mathrm{~Hz}$. Initial contact and toe off will be determined using a $50 \mathrm{~N}$ force plate threshold. All walking trials will be normalized to $100 \%$ of stance before being averaged for statistical analysis. Hip and knee joint angles, moments and excursions will be evaluated between limbs in both the sagittal and frontal plane.

Clinical outcome measures will include quadriceps strength index, single-legged hop test measures and patient reported outcome measure. Quadriceps strength will be measured using a maximal voluntary isometric contraction (MVIC) with a burst superimposition technique [49]. Activation deficits and isometric quadriceps strength will be measured using an electromechanical dynamometer (KIN-COM, Chattanooga Corp., Chattanooga, TN). Patients will be seated in an upright position with the hip and knee flexed to 90 degrees. Testing will be completed on the uninvolved limb followed by the involved limb. A quadriceps index (QI) will be calculated as the quotient of the involved quadriceps MVIC to the uninvolved quadriceps MVIC multiplied by 100 . The single-legged hop test measures [50] will consist of four hop tests in which each test will be administered as the uninvolved limb followed by the involved limb for the single hop for distance, crossover hop for distance, the triple hop for distance and 6-meter timed hop tests. A limb symmetry index (LSI) will be calculated from the average of two trials as the involved limb hop distance divided by the uninvolved limb hop distance multiplied by 100 . The 6 -meter timed hop will be calculated as the uninvolved limb hop time divided by the involved limb hop time multiplied by 100 . Patient reported outcome measures will be completed after all objective clinical measures have been collected. The Knee Outcome Survey-Activities of Daily Living Score (KOS-ADLS) and the Global Rating Scale of Perceived Knee Function (GRS) will be used to determine the patients perceived knee function. A strict return to sport criteria, established by Fitzgerald et al. [38], requires the patient to achieve $\geq$ $90 \%$ on the following measures: QI, all 4 single-legged hop tests, KOS-ADL's and GRS. Patients will be required to meet these criteria after training to allow for progressive return to sport activities. If patients do not meet these criteria after training they will be repeatedly tested every 2 4 weeks until all measures are met prior to returning to sport activity. The ACL-Return to Sport after Injury (ACL-RSI) has been validated to measure fear in patients after ACLR. This patient reported outcome measure will be used to objectively measure patient fear in the short to medium term (6 months -2 years).

\section{Joint loading}

Electromyography (EMG)-driven musculoskeletal modeling will be used to estimate muscle forces from EMG muscle data during walking trials [51]. Anatomical modeling of the pelvis and lower limbs will be scaled initially for each subject. The model will then be calibrated based on muscle parameters used to determine the EMG-to-force relationship. Through iterative adjustments, the muscle 


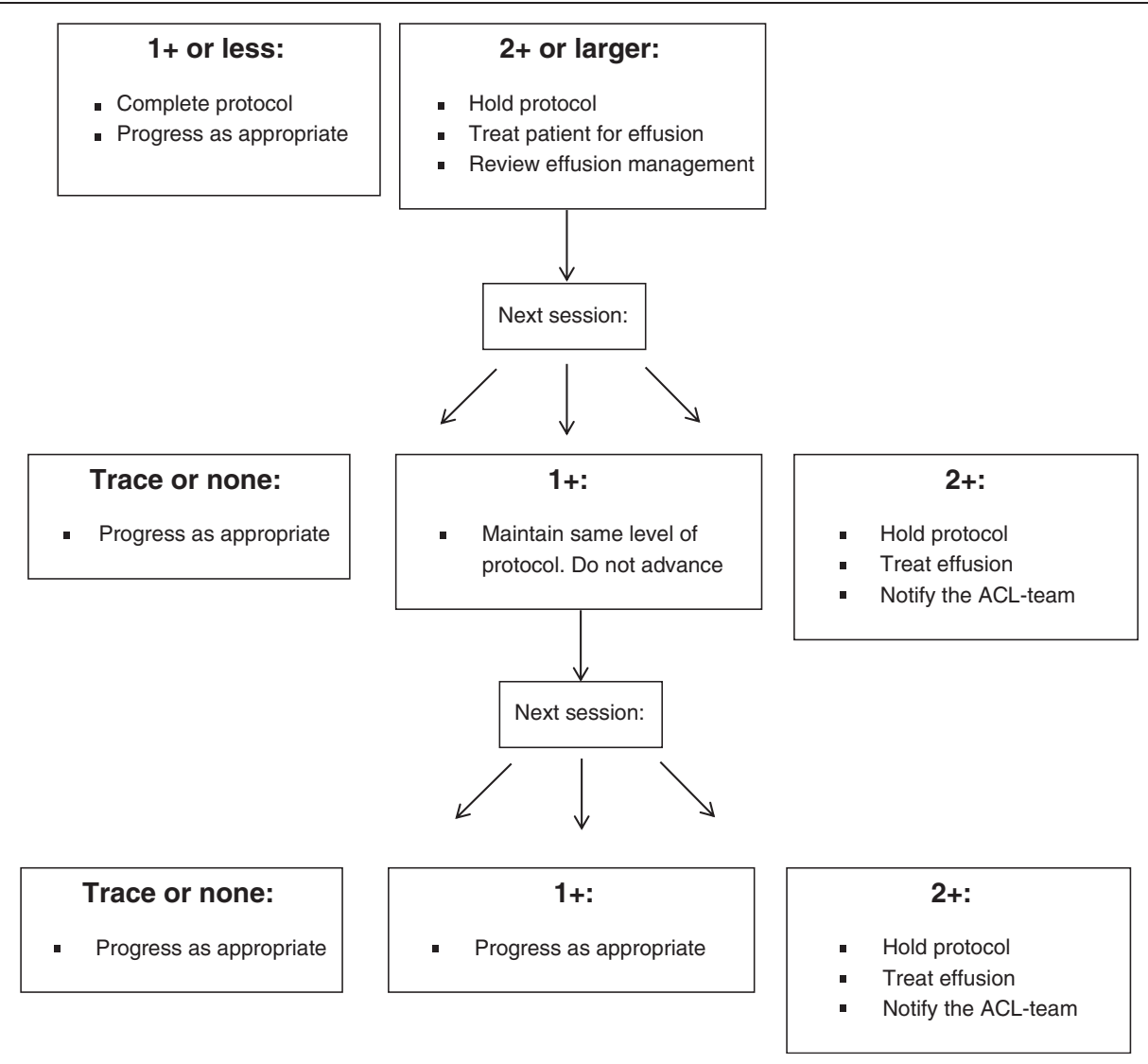

Figure 3 ACL-SPORTS training effusion protocol.

parameters will result in strong agreement with the sagittal plane net moments calculated from forward and inverse dynamics. Once the ideal model is determined, the muscle forces will be predicted from mathematical calculations from recorded EMG for three walking trials and

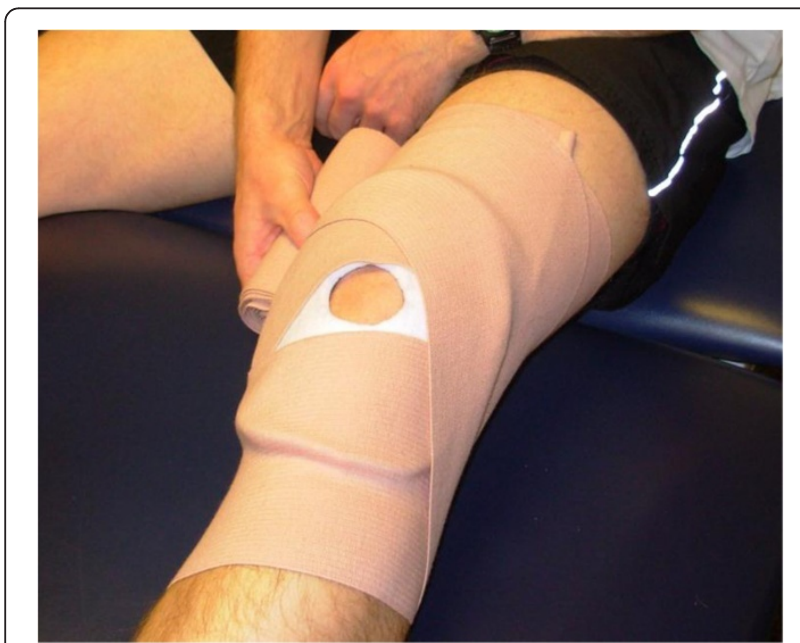

Figure 4 Donut with compression wrap for effusion management. converted to muscle force. A frontal plane moment balancing algorithm [52] will be used to calculate medial and lateral compartment contact forces. The knee adduction moment will be calculated using inverse dynamics and will be expressed about each contact point in the medial and lateral compartments. A balance of contact forces and muscle forces at each contact point will be summated to express the contact forces in the each compartment as well as the total joint forces.

\section{Secondary outcome measures}

Return to sport rates, re-injury rates and return to preinjury level of activity rates will be evaluated 1 and 2 years after surgery. Electromyography measures will be collected simultaneously with gait variables and will be used to further analyze muscle timing, co-contraction and activation patterns before and after the intervention as well as 1 and 2 years after surgery. IKDC 2000, Tampa Scale of Kinesiophobia (TSK-11), Knee Injury and Osteoarthritis Outcome Score (KOOS) including all 5 subsets and the Marx Activity Rating Scale (MARS) are additional patient reported outcome measures that will be collected at all-time points. 


\section{Sample size}

Minimal clinically importance differences (MCID) for sagittal plane gait variables have previously been established [18]. A power analysis with $\beta=0.20, \alpha=0.05$ and a medium effect size (0.3) determined that 72 subjects would be needed to detect differences between groups based on MCID's. To account for a $10 \%$ patient drop out a total of 80 subjects will be enrolled in this study. Forty patients will be in each group dichotomized by gender.

\section{Data and statistical analysis}

Differences between groups will be analyzed using an analysis of covariance (ANCOVA) for biomechanical gait measures and an analysis of variance (ANOVA) will be used for clinical variables. Group assignment will be blinded to the researcher using $A$ and $B$ variables. Assumptions of ANOVA testing will be confirmed prior to statistical analysis. Training group randomization will be used as the between-subjects factor with a within-subjects factor of time. A significance level of $\mathrm{p}<0.05$ will be set a priori.

\section{Timeline}

Human subjects review board approval was obtained in July 2011 from the University of Delaware Institutional Review Board and recruitment and training was initiated in November 2011. A projected 25 patients will be enrolled in the study within the first year followed by 30 and 25 patients respectively in the subsequent years. Final enrollment is planned to be completed by November 2014 and final data collection and analysis is planned to be completed by November 2016.

\section{Discussion}

Both short and long term outcomes after ACLR are poorer than previously reported in high-level athletes [1-5]. The explanation of these low return to sports rates appears to be multi-factorial, but may be heavily influenced by lower perceived level of knee function and fear of re-injury $[37,53,54]$. The relationship of physical performance measures to these subjective evaluations and perceptions of ability are unknown. The aim of this project is to compare the outcomes of two different return to sport training programs in order to establish best-practice guidelines for this high-risk population.

Initial ACL injury rates continue to be elevated and subsequent re-injury rates are even higher despite the positive evolution of post-operative rehabilitation protocols [4,23-28]. Quadriceps weakness, abnormal movement patterns and decreased knee function persist after athletes have returned to sports, supporting the need for a bilateral, neuromuscular training program to promote improved outcomes after ACLR [4,9-17]. Our program was compiled from the latest evidence emphasizing prevention exercises, quadriceps strengthening and perturbation training as a plausible mechanism by which clinicians can maximize post-operative function and reduce second ACL injury risk.

Our study is the first randomized control trial to evaluate the effects of a post-operative intervention program on joint loading, gait biomechanics and clinical outcome measures. Implementing this program in our physical therapy clinic with therapists who have years of expertise executing research protocols allows us to make this post-operative training program generalizable to clinical practice while maintaining the rigor of scientific research. Our subjects will represent several different orthopedic surgeons with a variety of graft types which will allow us to evaluate additional factors outside of our rehabilitation protocol. Our criterion to implement training is based on an array of evidence based clinical measures rather than time based measures (i.e. 6 months) or surgical findings (i.e. bone bruise, meniscus repair) [29,30,38,55]. Group randomization by gender will ensure that effects of treatment are adequately captured. Blinding of researchers collecting these data allows for unbiased reporting of results.

Through this ACL-SPORTS Training program we will be able to better evaluate the effects of neuromuscular training after surgery on knee joint loading, gait biomechanics and clinical outcome measures for these athletes. These variables are modifiable factors reported in the literature and most commonly utilized in clinical practice. Results of this study will allow us to develop future treatment plans to maximize functional outcomes in the short and long term after ACLR.

\section{Additional file}

Additional file 1: ACL-SPORTS Training. Treatment Procedural Checklist.

\section{Abbreviations}

ACL: Anterior cruciate ligament; ACLR: Anterior cruciate ligament reconstruction; IKDC 2000: International knee documentation committee 2000; PERT: Perturbation; ACL-SPORTS: Anterior cruciate ligament- specialized post-operative return-to-sports; STND: Standard; MVIC: Maximal voluntary isometric contraction; QI: Quadriceps index; LSI: Limb symmetry index; KOSADLS: Knee outcome survey-activities of daily living score; GRS: Global rating scale; ACL-RSI: Anterior cruciate ligament- return to sports after injury; EMG: Electromyography; TSK-11: Tampa scale of Kinesiophobia; KOOS: Knee injury and Osteoarthritis outcome score; MARS: Marx activity rating scale; MCID: Minimal clinically important differences; ANCOVA: Analysis of covariance; ANOVA: Analysis of variance.

\section{Competing interests}

The authors declare that they have no competing interests.

\section{Authors' contributions}

KW participated in developing the protocol, instructing the physical therapy staff on the treatment protocol, patient recruitment, research testing, contributed to trial registration on ClinicalTrials.gov, and drafted and critically revised this manuscript for important intellectual content. SLD contributed to the original idea of the study, developed a manual of operating procedures and critically revised this manuscript for important intellectual content. AHS participated in patient recruitment, procedural reliability, edited the treatment 
protocol, edited the procedural reliability forms and critically revised this manuscript for important intellectual content. LSM is the primary investigator of the randomized control trial and is responsible for the original idea of the study, registering the study on ClinicalTrials.gov, and critically revised this manuscript for important intellectual content. All authors read and approved the final manuscript.

\section{Authors' information}

KW, PT, DPT: KW is a PhD student and research assistant at the University of Delaware in the Biomechanics and Movement Science program. She is also a teaching assistant for several classes in the Department of Physical Therapy and is a per diem physical therapist at the University of Delaware Physical Therapy Clinic. SLD, PhD, PT: SLD is currently conducting research on the recovery of function in athletes with ACL injuries and femoroacetabular impingement (FAI). She is currently funded by the Sports Physical Therapy Section of the American Physical Therapy Association for her work with FAI. SLD is also a part-time physical therapist at the Ohio State University Sports Medicine Physical Therapy Clinic.

AHS, PT, DPT, OCS, SCS, ATC: AHS is a senior staff physical therapist in the Sports and Orthopaedic Physical Therapy Clinic as the University of Delaware. She is the physical therapy liaison overseeing the execution of the treatment protocol and completing procedural reliability for this study. LSM, PT, SCD, ATC, FAPTA: LSM, an alumni distinguished professor at the University of Delaware, is faculty in the Physical Therapy Department Kinesiology and Applied Physiology Department, Biomedical Engineering Department and the Biomechanics and Movement Science program. She is also the Academic Director of the University of Delaware Physical Therapy Clinic and the Director of the Residency Programs of the Physical Therapy Clinic at the University of Delaware.

\section{Acknowledgements}

This study is being funded by the National Institute of Health (5R01AR048212-07). The authors would like to acknowledge the ongoing assistance of the University of Delaware Physical Therapy Clinic staff for their role in implementing this study protocol as well as the Research Core at the University of Delaware for their contribution to this study.

\section{Author details}

'University of Delaware, Biomechanics and Movement Science Program, Newark, DE, USA. ${ }^{2}$ The Ohio State University, Sports Health and Performance Institute, Columbus, OH, USA. ${ }^{3}$ University of Delaware, Physical Therapy Department, Newark, DE, USA.

\section{Received: 22 February 2013 Accepted: 13 March 2013}

Published: 23 March 2013

\section{References}

1. Daniel DM, Lou SM, Bobson B, Fithian D, Rossman D, Kaufman K: Fate of the ACL-injured patient. A prospective outcome study. Am J Sports Med 1994, 22:632-644.

2. Lohmander LS, Ostenberg A, Englund M, Roos H: High prevalence of knee osteoarthritis, pain, and functional limitations in female soccer players twelve years after anterior cruciate ligament injury. Arthritis Rheum 2004 50:3145-3152

3. Myklebust $\mathrm{G}$, Bahr R: Return to play guidelines after anterior cruciate ligament surgery. Br J Sports Med 2005, 39:127-131.

4. Paterno MV, Schmitt LC, Ford KR, Rauh MJ, Myer GD, Huang B, Hewett TE: Biomechanical measures during landing and postural stability predict second anterior cruciate ligament injury after anterior cruciate ligament reconstruction and return to sport. Am J Sports Med 2010, 38:1968-1978.

5. Ardern $\mathrm{CL}$, Webster KE, Taylor NF, Feller Ja: Return to sport following anterior cruciate ligament reconstruction surgery: a systematic review and meta-analysis of the state of play. Br J Sports Med 2011, 45:596-606

6. George MS, Dunn WR, Spindler KP: Current concepts review: revision anterior cruciate ligament reconstruction. Am J Sports Med 2006, 34:2026-2037.

7. Weiler A, Schmeling A, Stöhr I, Kääb MJ, Wagner M: Primary versus singlestage revision anterior cruciate ligament reconstruction using autologous hamstring tendon grafts: a prospective matched-group analysis. Am J Sports Med 2007, 35:1643-1652.
8. Mayr R, Rosenberger R, Agraharam D, Smekal V, El Attal R: Revision anterior cruciate ligament reconstruction: an update. Arch Orthop Trauma Surg 2012, 132:1299-1313.

9. De Jong SN, Van Caspel DR, Van Haeff MJ, Saris DBF: Functional assessment and muscle strength before and after reconstruction of chronic anterior cruciate ligament lesions. Arthroscopy 2007, 23:21-28. 28.e1-3.

10. Eitzen I, Holm I, Risberg MA: Preoperative quadriceps strength is a significant predictor of knee function two years after anterior cruciate ligament reconstruction. Br J Sports Med 2009, 43:371-376.

11. Schmitt LC, Paterno MV, Hewett TE: The impact of quadriceps femoris strength asymmetry on functional performance at return to sport following anterior cruciate ligament reconstruction. J Orthop Sports Phys Ther 2012, 42:750-759.

12. Hartigan $E H$, Axe MJ, Snyder-Mackler L: Perturbation training prior to ACL reconstruction improves gait asymmetries in non-copers. $J$ Orthop Res 2009, 27:724-729.

13. Paterno MV, Ford KR, Myer GD, Heyl R, Hewett TE: Limb asymmetries in landing and jumping 2 years following anterior cruciate ligament reconstruction. Clin J Sport Med 2007, 17:258-262.

14. Roewer BD, Di Stasi SL, Snyder-Mackler L: Quadriceps strength and weight acceptance strategies continue to improve two years after anterior cruciate ligament reconstruction. J Biomech 2011, 44:1948-1953.

15. Castanharo R, Da Luz BS, Bitar AC, D'Elia CO, Castropil W, Duarte M: Males still have limb asymmetries in multijoint movement tasks more than 2 years following anterior cruciate ligament reconstruction. J Orthop Sci 2011, 16:531-535.

16. Myer GD, Martin L, Ford KR, Paterno MV, Schmitt LC, Heidt RS, Colosimo A, Hewett TE: No association of time from surgery with functional deficits in athletes after anterior cruciate ligament reconstruction: evidence for objective return-to-sport criteria. Am J Sports Med 2012, 40:2256-2263.

17. Logerstedt D, Lynch A, Axe MJ, Snyder-Mackler L: Symmetry restoration and functional recovery before and after anterior cruciate ligament reconstruction. Knee Surg Sports Traumatol Arthrosc 2012. PMID: 22349604.

18. DiStasi SL, Snyder-Mackler L: The effects of neuromuscular training on the gait patterns of ACL-deficient men and women. Clin Biomech (Bristol, Avon) 2012, 27:360-5.

19. Myer GD, Brent JL, Ford KR, Hewett TE: Real-time assessment and neuromuscular training feedback techniques to prevent anterior cruciate ligament injury in female athletes. Strength Cond J 2011, 33:21-35.

20. Laboute E, Savalli L, Puig P, Trouve P, Sabot G, Monnier G, Dubroca B: Analysis of return to competition and repeat rupture for 298 anterior cruciate ligament reconstructions with patellar or hamstring tendon autograft in sportspeople. Ann Phys Rehabil Med 2010, 53:598-614.

21. Salmon L, Russell V, Musgrove T, Pinczewski L, Refshauge K: Incidence and risk factors for graft rupture and contralateral rupture after anterior cruciate ligament reconstruction. Arthroscopy 2005, 21:948-57.

22. Van Eck CF, Schkrohowsky JG, Working ZM, Irrgang JJ, Fu FH: Prospective analysis of failure rate and predictors of failure after anatomic anterior cruciate ligament reconstruction with allograft. Am J Sports Med 2012, 40:800-7.

23. Paterno MV, Rauh MJ, Schmitt LC, Ford KR, Hewett TE: Incidence of contralateral and ipsilateral anterior cruciate ligament (ACL) injury after primary ACL reconstruction and return to sport. Clin I Sport Med 2012, 22:116-21.

24. Shelbourne KD, Gray T, Haro M: Incidence of subsequent injury to either knee within 5 years after anterior cruciate ligament reconstruction with patellar tendon autograft. Am J Sports Med 2009, 37:246-51.

25. Barber-Westin SD, Noyes FR: Objective criteria for return to athletics after anterior cruciate ligament reconstruction and subsequent reinjury rates: a systematic review. Phys Sportsmed 2011, 39:100-10.

26. Leys T, Salmon L, Waller A, Linklater J, Pinczewski L: Clinical results and risk factors for reinjury 15 years after anterior cruciate ligament reconstruction: a prospective study of hamstring and patellar tendon grafts. Am J Sports Med 2012, 40:595-605.

27. Wright RW, Magnussen RA, Dunn WR, Spindler KP: Ipsilateral graft and contralateral $\mathrm{ACL}$ rupture at five years or more following $\mathrm{ACL}$ reconstruction: a systematic review. J Bone Joint Surg Am 2011, 93:1159-65.

28. Bourke HE, Salmon LJ, Waller A, Patterson V, Pinczewski La: Survival of the anterior cruciate ligament graft and the contralateral $\mathrm{ACL}$ at a minimum of 15 years. Am J Sports Med 2012, 40:1985-92. 
29. Adams D, Logerstedt D, Hunter-Giordano A, Axe MJ, Snyder-Mackler L: Current concepts for anterior cruciate ligament reconstruction: a criterion-based rehabilitation progression. J Orthop Sports Phys Ther 2012, 42:601-14.

30. Myer GD, Paterno MV, Ford KR, Quatman CE, Hewett TE: Rehabilitation after anterior cruciate ligament reconstruction: criteria-based progression through the return-to-sport phase. J Orthop Sports Phys Ther 2006, 36:385-402.

31. Myer GD, Paterno MV, Ford KR, Hewett TE: Neuromuscular Training Techniques to Target Deficits Before Return to Sport After Anterior Cruciate Ligament Reconstruction. J Strength Cond Res 2008, 22:987-1014

32. Wilk KE, Macrina LC, Cain EL, Dugas JR, Andrews JR: Recent advances in the rehabilitation of anterior cruciate ligament injuries. J Orthop Sports Phys Ther 2012, 42:153-71.

33. Hartigan EH, Axe MJ, Snyder-Mackler L: Time line for noncopers to pass return-to-sports criteria after anterior cruciate ligament reconstruction. J Orthop Sports Phys Ther 2010, 40:141-54.

34. Ardern CL, Webster KE, Taylor NF, Feller Ja: Return to the preinjury level of competitive sport after anterior cruciate ligament reconstruction surgery: two-thirds of patients have not returned by 12 months after surgery. Am J Sports Med 2011, 39:538-43.

35. Webster KE, Feller JA, Lambros C: Development and preliminary validation of a scale to measure the psychological impact of returning to sport following anterior cruciate ligament reconstruction surgery. Phys Ther Sport 2008, 9:9-15.

36. McCullough KA, Phelps KD, Spindler KP, Matava MJ, Dunn WR, Parker RD, Reinke EK: Return to high school- and college-level football after anterior cruciate ligament reconstruction: A multicenter orthopaedic outcomes network (MOON) cohort study. Am J Sports Med 2012, 40:2523-9.

37. Webster KE, Feller JA, Lambros C: Development and preliminary validation of a scale to measure the psychological impact of returning to sport following anterior cruciate ligament reconstruction surgery. Phys Ther Sport 2008, 9:9-15.

38. Fitzgerald GK, Axe MJ, Snyder-Mackler L: The efficacy of perturbation training in nonoperative anterior cruciate ligament rehabilitation programs for physically active individuals. Phys Ther 2000, 80:128-40.

39. Fitzgerald GK, Axe M, Snyder-mackler L: Proposed practice guidelines for non operative anterior cruciate ligament rehabilitation of physically active individuals. J Orthop Sports Phys Ther 2000, 30:194-203.

40. Boutron I, Moher D, Altman DG, Schulz KF, Ravaud P: Extending the CONSORT statement to randomized trials of nonpharmacologic treatment: explanation and elaboration. Ann Intern Med 2008, 148:295-310.

41. Sturgill LP, Snyder-Mackler L, Manal TJ, Axe MJ: Interrater reliability of a clinical scale to assess knee joint effusion. J Orthop Sports 2009, 39:845-9.

42. Myer GD, Ford KR, Hewett TE: Methodological approaches and rationale for training to prevent anterior cruciate ligament injuries in female athletes. Scand J Med Sci Sports 2004, 14:275-85.

43. Hewett TE, Stroupe AL, Nance TA, Noyes FR: Plyometric training in female athletes: decreased impact forces and increased hamstring torques. Am J Sports Med 1996, 24:765-772.

44. Hewett TE, Lindenfeld TN, Riccobene JV, Noyes FR: The effect of neuromuscular training on the incidence of knee injury in female athletes. A prospective study. Am J Sports Med 1999, 27:699-706.

45. Heitkamp H, Horstmann T, Mayer F, Weller J, Dickhuth H: Gain in strength and muscular balance after balance training. Int J Sports Med 2001, 22:285-290

46. Knapik J, Bauman C, Jones B, Harris J, Vaughan L: Preseason strength and flexibility imbalances associated with athletic injuries in female collegiate athletes. Am J Sports Med 1991, 19:76-81.

47. Lehnhard RA, Lehnhard HR, Young R, Butterfield SA: Monitoring Injuries on a College Soccer Team: The Effect of Strength Training. J Strength Cond 1996, 10:115-119.

48. Myer GD, Ford KR, Palumbo JP, Hewett TE: Neuromuscular Training Improved Performance and Lower-Extremity Biomechanics in Female Athletes. J Strength Cond Res 2005, 19:51-60.

49. Snyder-Mackler L, Delitto A, Stralka SW, Bailey SL: Use of electrical stimulation to enhance recovery of quadriceps femoris muscle force production in patients following anterior cruciate ligament reconstruction. Phys Ther 1994, 74:901-7.
50. Noyes FR, Barber SD, Mangine RE: Abnormal Lower Limb Symmetry Determined By Function Hop Tests After ACL Rupture. Am J Sports Med 1991, 19:513-518.

51. Buchanan TS, Lloyd DG, Manal K, Besier TF: Neuromusculoskeletal modeling: estimation of muscle forces and joint moments and movements from measurements of neural command. J Appl Biomech 2004, 20:367-95.

52. Winby CR, Lloyd DG, Besier TF, Kirk TB: Muscle and external load contribution to knee joint contact loads during normal gait. J Biomech 2009, 42:2294-300.

53. Logerstedt D, Lynch A, Axe MJ, Snyder-Mackler L: Pre-operative quadriceps strength predicts IKDC2000 scores 6 months after anterior cruciate ligament reconstruction. Knee 2012. PMID: 23022031

54. Logerstedt D, Grindem H, Lynch A, Eitzen I, Engebretsen L, Risberg MA, Axe MJ, Snyder-Mackler L: Single-Legged Hop Tests as Predictors of SelfReported Knee Function After Anterior Cruciate Ligament Reconstruction: The Delaware-Oslo ACL Cohort Study. Am J Sports Med 2012, 40:2348-56.

55. Thomeé R, Kaplan Y, Kvist J, Myklebust G, Risberg MA, Theisen D, Tsepis E, Werner S, Wondrasch B, Witvrouw E: Muscle strength and hop performance criteria prior to return to sports after $\mathrm{ACL}$ reconstruction. Knee Surg Sports Traumatol Arthrosc 2011, 19:1798-805.

doi:10.1186/1471-2474-14-108

Cite this article as: White et al:: Anterior cruciate ligament- specialized post-operative return-to-sports (ACL-SPORTS) training: a randomized control trial. BMC Musculoskeletal Disorders 2013 14:108.

\section{Submit your next manuscript to BioMed Central and take full advantage of:}

- Convenient online submission

- Thorough peer review

- No space constraints or color figure charges

- Immediate publication on acceptance

- Inclusion in PubMed, CAS, Scopus and Google Scholar

- Research which is freely available for redistribution 